\title{
Current trends in electrochemical studies of nonaqueous solutions
}

\author{
J. F. Coetzee \\ Department of Chemistry, University of Pittsburgh, Pittsburgh, Pennsylvania \\ 15260, USA
}

\begin{abstract}
Current trends in electrochemical studies of nonaqueous solutions are discussed with the emphasis on experimental approaches judged to be particularly promising for the future development of both fundamental and applied aspects of the field of solution chemistry. Topics include recent developments in the application of potentiometric sensors to nonaqueous solutions, the potentialities of such new materials as reactively sputtered iridium oxide as hydrogen ion sensors, and the demonstrated or potential advantages of microelectrodes with diameters less than approximately $20 \mu \mathrm{m}$ in cyclic voltammetry and a variety of other electrochemical techniques applicable to nonaqueous solutions. The extension of electrochemical measurements to nonpolar media is emphasized. Recent developments in certain other areas such as applications of solid electrolytes and chemically modified electrodes, and studies of interfaces between immiscible solvents, are briefly discussed.
\end{abstract}

\section{INTRODUCTION}

Electrochemistry is a classical field, but it is currently developing rapidly owing largely to improvements in electronics and the introduction of new materials having electronic or ionic conductivity. In some of these developments nonaqueous solvents are used as a matter of necessity, e.g., in lithium batteries. On the other hand, many interesting developments so far have been limited to aqueous solutions even though no fundamental reasons exist why extension to nonaqueous solutions should not be feasible. In this respect current developments follow the historical pattern of the field of electrochemistry in that applications to nonaqueous media generally lag far behind those involving aqueous solutions. Reasons undoubtedly include experimental difficulties encountered in the more interesting nonaqueous solvents and the complexity of ion-ion and ion-solvent interactions in such solvents, particularly those that have weak solvating abilities and/or low permittivities.

No attempt is made here to review all recent developments in the electrochemistry of nonaqueous solutions. Rather, a limited number of examples of approaches judged to be particularly promising for the development of the field of nonaqueous solution chemistry are presented.

\section{APPLICATIONS OF POTENTIOMETRIC SENSORS IN NONAQUEOUS SOLUTIONS}

A potentiometric ion sensor has the major merit that, in any solvent in which it functions properly, it measures the activity (hence, the reactivity) of the "free" ion, even when a large excess of "bound" ion (electrostatically bound ion pairs or higher aggregates, covalently bound complexes, etc.) is present, thereby providing information that of ten is not accessible to other probes. The result is that potentiometric sensors have been widely used in many polar solvents for analytical purposes (either in direct potentiometry, e.g., in measuring hydrogen ion activity, or in potentiometric titrations), and in the determination of such fundamental thermodynamic or pseudo-thermodynamic quantities as equilibrium constants or free energies of transfer of ions from one solvent to another.

\section{General applications in nonaqueous solutions}

An obvious requirement is that the sensor material should be unreactive and insoluble in the test solution, which generally rules out the use in nonaqueous solvents of ion selec- 
tive electrodes (ISEs) with liquid membranes. Solid state and glass ISEs as well as other types of potentiometric sensors, such as indicator electrodes of the first kind (e.g., Ag) and the second kind (e.g., $\mathrm{Ag} / \mathrm{AgCl})$, can be used in a wide variety of solvents.

When glass electrodes and possibly also certain other ISEs are exposed to aqueous solutions, gel layers form on the membrane surfaces. It is generally accepted that the membrane (boundary) potentials of such electrodes are established by ion exchange processes occurring in the gel layers. The literature contains scattered reports that these electrodes malfunction in nonaqueous solutions owing to dehydration (hence, deactivation) of the gel layers. We (and others) have not encountered such problems, except after leaving the ISE in the nonaqueous solvent for unnecessarily long times (conditioning of the electrode for 1-2 h generally suffices) or when the electrode is used in exceptionally reactive solutions, e.g., perchloric acid in sulfolane (ref. 1).

The literature also contains many reports of non-Nernstian response of ISEs in unbuffered nonaqueous solutions, as well as of limited dynamic range in such solutions, e.g., lower detection limits of typically $10^{-4} \mathrm{M}$, as compared to $10^{-5}$ to $10^{-7} \mathrm{M}$ in aqueous solution. We attribute these observations to the presence of impurities that lower the activity of the sensed ion (ref. 2); this important matter is discussed in the next section. In buffered solutions these problems are less severe.

A comprehensive review of applications of ISEs in nonaqueous solutions has been published under IUPAC auspices by Pungor et al. (ref. 3). A few representative examples are these. (a) Glass ISE for hydrogen ion: Numerous examples of potentiometric titrations, determination of dissociation constants of Brønsted acids and other studies of proton transfer reactions in alcohols and dipolar aprotic solvents as well as their mixtures with water. It is to be noted that the hydrogen electrode also functions satisfactorily in many, but not all, of these solvents. (b) Glass ISEs for alkali metal ions: Evaluation of free energies of transfer of alkali metal ions among alcohols and dipolar aprotic solvents. Determination of formation constants of complexes with macrocyclic ligands. (c) Solid state ISEs for copper(II), fluoride and other ions: Studies of complexation (e.g., Cu ${ }^{2+}+$ EDTA) and precipitation (e.g., $\mathrm{La}^{3+}+\mathrm{F}^{-}$) in alcohols and dipolar aprotic solvents.

\section{Applications to characterization of reactive impurities in nonaqueous solvents}

A specific recent application of potentiometric sensors, particularly ISEs, is in the characterization of reactive impurities in nonaqueous solvents - a matter of crucial importance throughout the broad field of solution chemistry.

All chemicals contain impurities, but in the case of solvents, which out of necessity must be used in high concentrations, interference by impurities can be particularly severe. This is especially true for solvents that are relatively inert (in the sense of being weak solvators), e.g., sulfolane, propylene carbonate, acetonitrile and thiony1 chloride. This serious problem is further compounded by a number of factors. (a) The nature and concentrations of impurities in a given solvent frequently vary with the source and even the batch of solvent. Manufaturers of ten change sources of raw materials as well as synthetic methods and thereby introduce different impurities. (b) Typical reagent grades of solvents contain reactive impurities in sufficiently high concentrations to compromise certain applications of the solvent. (c) Important reactive impurities (e.g., amines in alcohols and dipolar aprotic solvents) in the past have escaped detection by such probes as gas chromatography (see below). (d) "Standard" purification procedures of ten fail to lower the concentrations of such impurities to acceptable levels, so that new purification procedures tailored to the intended use of the solvent must be developed. These important matters are discussed in this section.

A few examples of interferences caused by impurities in nonaqueous solutions are given in Table 1. While it is obvious that impurity effects generally will be most severe in dilute solutions and in studies concerned with reactivities of solutes, interferences may be far more subtle (e.g., catalytic effects - information on this problem is just beginning to appear) or may occur even in concentrated solutions (e.g., in the 1 to $2 \mathrm{M}$ solutions of electrolytes used in lithium batteries). In fact, problems encountered with lithium batteries provide good examples of the more subtle types of impurity effects. The most obvious effect to be expected from protic impurities is of course hydrogen evolution and (in the case of water) precipitation of a film of lithium oxide or hydroxide (which are extremely insoluble in aprotic solvents) on the lithium electrode. However, it is not yet obvious how this type of film would affect overall battery performance. It would impart some degree of passivity to the lithium surface, which could be desirable if it prevents extensive reaction of lithium metal with the aprotic solvent (which is thermodynamically favored). In primary batteries, then, such a film would be beneficial provided that it allows adequate lithium ion transport while the battery is operating under load. In secondary, rechargeable batteries, however, it would create serious problems if it insulates electrodeposited lithium metal from the substrate; this problem has been thoroughly discussed (ref.4). In fact, the ideal film should possess a very 
TABLE 1. Interference by impurities in important applications of nonaqueous solvents

Application

Interference

Studies of reactivities of solutes and mechanisms of reactions: widespread currently and in the past, especially in organic and organometallic chemistry

Use of highly reactive reagents: new types of chemistry. Examples: superacids ( $\mathrm{HClO}_{4}$ in SL), superbases (Cs dimsyl in DMSO)

Taking advantage of extended electrochemical "windows" of certain nonaqueous solvents: new types of electron transfer reactions (e.g., in organometallic chemistry)

Commercial applications, e.g., high energy density batteries: $\mathrm{Li} / 1 \mathrm{M} \mathrm{LiAsF}_{6}$ in $\mathrm{PC} / \mathrm{TiS}_{2}$
May drastically affect thermodynamics and kinetics, and even mechanisms, of reactions

Lowers the reactivities, therefore limits the utility, especially of dilute solutions

a. Limits accessible potential range

b. Increases background current, decreases S/N

c. Catalyzes undesirable reactions

a. Corrodes Li anode

b. Catalyzes degradation of aprotic solvent

c. May affect discharge mechanism of cathode

particular balance of coherence, proper morphology, and proper electronic and ionic properties. Impurities may also have other, less obvious, effects. For example, it appears from the thorough studies by Pons (ref. 5) of reduction processes occurring at a platinum electrode in acetonitrile at potentials near the lithium potential that hydride ion can be produced if some water is present, and that this has profound effects on the interaction of acetonitrile with the electrode. As another example, it is conceivable that protic impurities may dramatically influence the discharge mechanisms of such cathode materials as $\mathrm{FeS}_{2}, \mathrm{MnO}_{2}$ and $\mathrm{CF}_{\mathrm{x}}$.

It is clear from these examples that, for many applications of nonaqueous solvents, what is really needed is to routinely characterize impurities on the basis of their reactivities toward appropriately chosen probes, rather than on the basis of their concentrations only. Potentiometric sensors can provide such information, as we have shown in ref. (2) from which we now quote the essential details. The approach involves adding such highly reactive ions as hydrogen, hydroxyl, copper(II), mercury(II), and fluoride as probes and monitoring their activities over a wide concentration range (typically some 7 decades) with the corresponding indicator electrodes, usually ISEs. Taking the indicator electrode for a metal cation $\mathrm{M}^{\mathrm{n}+}$ as an example, its potential as a function of "free" metal ion concentration [M] at constant ionic strength and in the absence of ion association or other complicating reactions is given by

$$
E=K+S \log [M]
$$

where the slope $S=0.0592 / \mathrm{n} \mathrm{V}$ at $298 \mathrm{~K}$ and the constant $\mathrm{K}$ contains, in addition to the electrode constant, also the liquid junction potential between the analyte and reference electrode solutions as well as the activity coefficient of $\mathrm{M}^{\mathrm{n}+}$. Now, if a 1igand $\mathrm{L}$ is present which forms complexes $\mathrm{ML}, \mathrm{ML}_{2}, \ldots, \mathrm{ML}_{\mathrm{n}}$ with the overall formation constants $R_{1}$, $\beta_{2}, \ldots, \beta_{n}$, then the free metal ion concentration will be related to the total metal ion concentration $C_{M}$ as in

$$
C_{M} /[M]=1+B_{1}[L]+\beta_{2}[L]^{2} \ldots+\beta_{n}[L]^{n}
$$

where [L] is the concentration of free $\mathrm{L}$ which in turn is related to the total ligand concentration $\mathrm{C}_{\mathrm{L}}$ as in eq 3 . Hence the potential of the indicator electrode as a function of

$$
C_{L}=[L]+[M L]+2\left[M L_{2}\right]+\ldots+n\left[M L_{n}\right]
$$

$C_{M}$ will deviate from that in the absence of $L$ (when $[M]=C_{M}$ ) by an amount $\Delta E$ given by eq 4. Consequent1y, when increasing concentrations of $\mathrm{M}^{\mathrm{n}+}$ are added to a solvent con- 


$$
\Delta E=-S \log \left(1+\beta_{1}[L]+\beta_{2}[L]^{2}+\ldots+\beta_{n}[L]^{n}\right)
$$

taining a constant concentration of $\mathrm{L}$, the plot of the potential of a properly responding indicator electrode for $\mathrm{M}^{\mathrm{n}+}$ vs. $\log \mathrm{C}_{\mathrm{M}}$ (essentially a titration curve but with $\mathrm{C}_{\mathrm{M}}$ varied over a sufficiently wide range) should show three distinct segments. These correspond to the regions in which, successively, (a) $C_{M}$ is so small that [L] $C_{L}$, so that $\Delta E$ has a constant nonzero value, (b) [L] varies, so that $\Delta \mathrm{E}$ varies, and (c) $C_{M}$ is so large that the terms containing [L] in eq 4 are negligibly small, so that $\Delta \mathrm{E}=0$. This behavior is illustrated by the calculated curve DCBA in Fig. 1. The region CB is characterized by a varying "super-Nernstian" response (relative to the response in the absence of $L$ ) and is of most analytical utility.

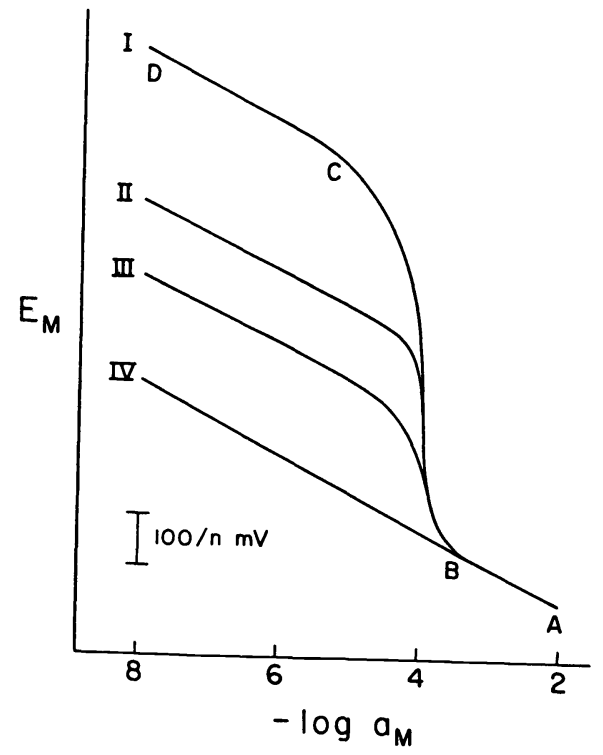

Figure 1

Calculated response of indicator electrode for $\mathrm{M}^{\mathrm{n}+}$ in the presence of $10^{-4}$ $M$ ligand L. (I) $\log \beta_{n}=10,18,24$ and 28 for $n=1,2,3$ and 4 ; example: $\mathrm{Hg}^{2+}$ + aliphatic amines in methanol. (II) $10 \mathrm{~g}$ $\beta_{1}=10$; example: $\mathrm{H}^{+}+$aliphatic amines in methano1. (III) $\log \beta_{n}=6,11,15$ and 18 for $n=1,2,3$ and 4 ; example: $\mathrm{Cu}^{2+}+$ aliphatic amines in methanol. (IV) No ligand present, slope $=59.2 / \mathrm{n}$ $\mathrm{mV} /$ decade in am. From ref. (2), by permission.

In this region, $\triangle E$ depends on $C_{M}, C_{L}$, and the formation constants, while in region $D C \Delta E$ is independent of $C_{M}$. In certain cases (close to point $B$ in the region $C B$ or over the whole "titration curve" with $\mathrm{H}^{+}, \mathrm{OH}^{-}$, or $\mathrm{F}^{-}$, rather than $\mathrm{M}^{\mathrm{n}}$, as the probe ion) often only 1:1 complexation occurs, so that eq 4 simplifies (and, in the case of $\mathrm{OH}^{-}$or $\mathrm{F}^{-}, \Delta E$ is positive, rather than negative). If, on the other hand, the ligand is multifunctional or more than one ligand is present, eq 4 becomes correspondingly more complex. The same is true when the ligand is present in a protonated form $\mathrm{H}_{\mathrm{q}} \mathrm{L}$, especially when the indicator electrode for $\mathrm{M}^{\mathrm{n}}$ also responds to the liberated hydrogen ions, which is the case for such sulfide-containing ISEs as that for the copper(II) ion in certain nonaqueous solvents.

It is to be noted that the approach described here is valid only if the indicator electrode is responding in a reproducible (preferably Nernstian) manner over a wide range of activities of the "free" probe ion, both in the absence and in the presence of the complexes of the probe ion. We have shown (ref. 2) that under proper experimental conditions (e.g., by using polished electrode membranes in flow systems) this is indeed the case.

An example of results obtained for methanol by means of this approach is shown in Fig. 2 . Values of $\Delta E$ (eq 4) indicated that impurities acting as strong ligands towards the copper(II) ion probe (probably amines) were present, and the location of point B in Fig. 2 showed that the total impurity concentration was ca. $10^{-4} \mathrm{M}$. It then became possible to optimize columns and experimental parameters for gas chromatographic analysis of the volatile and reactive amines (a difficult analytical problem) and to confirm the presence of $2 \times 10^{-4} \mathrm{M}$ trimethylamine.

By using other probe ions for which reliable ISEs, resistant to nonaqueous solvents, are available, other classes of reactive impurities can be characterized. For example, with the glass electrode for hydrogen ion, proton acceptors (i.e., substances competing successfully with the particular solvent for protons) can be detected and quantitated, while the same can be accomplished for proton as well as hydrogen bond donors with the fluoride ISE ( $\mathrm{LaF}_{3}$ single crystal membrane).

Since the value of $\Delta \mathrm{E}$ (eq 4) depends on the stability of the complexes formed by the probe ion, the approach described here has another important merit: its lower detection 1imit is 


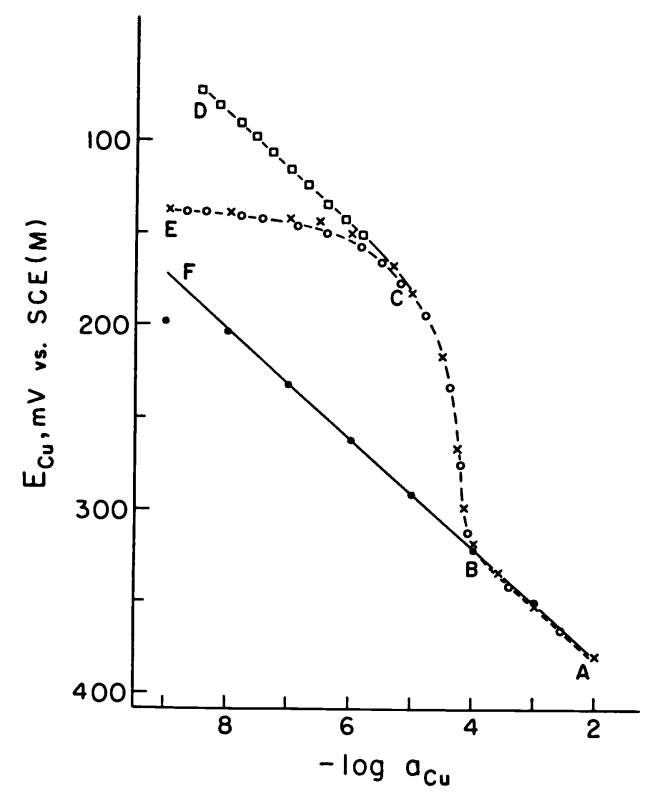

Figure 2

Response of copper(II) ion selective electrode in methanol. Open circles: observed response with Fisher Spectranalyzed methanol. Squares: calculated response based on complexation of copper(II) ion with amines. Crosses: calculated response based on complexation with amines as well as the presence of 3 $x 10^{-7} \mathrm{M}$ copper(II) ion also shown to be present as an impurity. Closed circles: observed response after appropriate purification of methanol. F: theoretical response in absence of impurities. From ref. (2), by permission.

self-adjusting in that it is lowest (most favorable) in the very solvents in which impurities are most harmful, i.e., relatively inert solvents. In such solvents, its lower detection limit can be as low as $10^{-7} \mathrm{M}(0.01 \mathrm{ppm})$, which is much lower than that attainable with other methods.

The approach described here so far has led to the detection and determination of previously undetected reactive impurities in typical reagent grades of several solvents (ref. 2, 6). These impurities include trimethylamine in methanol, triethylamine in ethanol, n-propylamine and pyridine in acetonitrile, and triethylamine in propylene carbonate. It seems likely that the amines in the alcohols originate from nitrogen present in the industrial grade reagents currently used in the synthesis of alcohols, e.g., $\mathrm{CO}+2 \mathrm{H}_{2}+$ $\mathrm{CH}_{3} \mathrm{OH}$ and $\mathrm{C}_{2} \mathrm{H}_{4}+\mathrm{H}_{2} \mathrm{O}+\mathrm{C}_{2} \mathrm{H}_{5} \mathrm{OH}$. (As supplies of petrochemicals dwindle, more and more ethanol may again be produced by fermentation, which introduces different impurities). Commercial acetonitrile is generally a byproduct of the large-scale acrylonitrile process, i.e., $2 \mathrm{H}_{2} \mathrm{C}=\mathrm{CH}-\mathrm{CH}_{3}+2 \mathrm{NH}_{3}+3 \mathrm{O}_{2} \rightarrow 2 \mathrm{H}_{2} \mathrm{C}=\mathrm{CH}-\mathrm{CN}$, while propylene carbonate is produced by reaction of propylene oxide with carbon dioxide, using as catalyst tetraethylammonium bromide which, in turn, is made from triethylamine and ethyl bromide. We suspect that many other solvents, those containing nitrogen as well as those that do not, contain amines as impurities.

We have described procedures that are effective in lowering the amine content of alcohols by two or three decades (ref. 2); more complete removal of amines is difficult. Procedures will be described for acetonitrile and propylene carbonate (ref. 6).

\section{Potentiometric sensors based on new materials}

The most promising example of this type is reactively sputtered iridium oxide which behaves as a hydrogen ion sensor. The ubiquitous glass electrode does meet most of the requirements for the ideal hydrogen ion sensor. Thus, the gel layer on its surface undergoes relatively rapid proton exchange with the solution, and its bulk phase is an ionic semiconductor without any electronic conductivity which would impart undesirable response to redox systems that may be present in solution. On the other hand, the glass electrode does suffer from interference by sodium and other univalent cations that can compete with protons for exchange sites. It also has limited low temperature capability (even when its internal aqueous solution is replaced by a nonaqueous solution) because its response becomes slow, and its ion exchanging gel layer is destroyed at high temperatures and in such hostile media as dilute hydrofluoric acid and highly acidic or basic solutions. As a result of these limitations, substitutes have been sought for a long time. Antimony oxide electrodes found some applications owing to their high temperature capability and relative freedom from cation interference, but they suffer from redox sensitivity to such substances as dissolved oxygen. Among other metal oxides, iridium oxide is particularly interesting. It has both electrochromic (ref. 7, 8) and hydrogen ion sensing (ref. 9-11) properties. The mechanism of its response to hydrogen ions has not yet been established. Like several other metal oxides (ref. 11), it has electronic conductivity because of a slight oxygen deficiency with a concomitant slightly lower effective oxida- 
tion state of the metal. For such oxides, Fogg and Buck (ref. 11) suggested several possible response mechanisms and proposed that the most likely is a single-phase oxygen

$$
\mathrm{MO}_{\mathrm{x}}+2 \delta \mathrm{H}^{+}+2 \mathrm{e}^{-} \neq \mathrm{MO}-\delta+\delta \mathrm{H}_{2} \mathrm{O}
$$

intercalation process as in eq (5), rather than a simple ion exchange process. The hydrogen ion response of iridium oxide does depend on the method used for its preparation, however. It can be made by chemical oxidation, by electrochemical oxidation, and by reactive sputtering from an iridium metal target in an oxygen plasma on a variety of substrates such as iridium or other metals, or ceramics. The (amorphous) sputtered material gives the best potentiometric response (ref. 10). Of particular potential significance for studies in nonaqueous solutions are the observations that the iridium oxide sensor responds faster than the glass electrode does and that it is resistant to such corrosive solutions as dilute hydrofluoric acid and $5 \mathrm{M}$ sulfuric acid. Applications to "pure" nonaqueous solutions have not yet been reported, but Bordi et al. (ref. 12) found that the sensor responds rapidly and in near-Nernstian manner in $1: 1$ mixtures of dimethy 1 sulfoxide with aqueous acetate, cacodylate and phosphate buffers over the $\mathrm{pH}$ range of 4.4 to 9.1 (determined spectrophotometrically) at temperatures of $+20,0$ and $-20{ }^{\circ} \mathrm{C}$.

It appears that the iridium oxide sensor for hydrogen ion holds significant promise for measurements in highly acidic or basic nonaqueous solutions over a wide temperature range.

\section{MICROELECTRODES}

Electrochemistry at microelectrodes is a recent development holding much promise, especially for nonaqueous solutions. The term "microelectrode" is reserved here for electrodes with diameters not greater than approximately $20 \mu \mathrm{m}$. Carbon fibers fall in this category; these are recently introduced materials used in a variety of consumer and other products for reasons of their high strength/weight ratio. The fibers are manufactured by pyrolysis of polymer textiles or pitch. Their surface composition is a matter of some controversy, but it appears that a variety of closely related oxygen-containing functional groups are present, including hydroxy1, carbony1, carboxy1 and ester groups.

Consequently, in electrochemical applications carbon fibers may be regarded as highly complex chemically modified electrodes. Such surface modification generally improves electron transfer. Suggested reasons include resonance tunnelling via acceptor sites formed on the surface electronic band gap, promotion of the transition state by changes in the characteristics of the electric double layer, and direct interaction of reactants with surface groups (ref. 13). A review of the first electroanalytical applications of carbon fibers appeared in 1985 (ref. 13). Metal (platinum and gold) fibers are also available in diameters down to $1 \mu \mathrm{m}$. The fibers are typically mounted at the tip of a capillary with epoxy adhesive, and are used in cylindrical or, more commonly, disc configuration (ref. 14).

Several advantages accrue from the use of such small electrodes. (a) They allow in vivo electrochemical measurements. For example, Wightman (ref. 15) has carried out voltammetric determinations of neurotransmitter molecules (e.g., dopamine) in rat brain. (b) Since currents in voltammetry at microelectrodes are sma11, iR-drops can be negligible even in highly resistive solutions. Thus, voltammetry becomes a viable technique even in such nonpolar solvents as hexane (ref. 16) or in polar solvents without added supporting electrolyte (ref. 14). This feature may be the single most significant advantage of microelectrodes and may lead to major expansion in the scope of the electrochemistry of organic compounds. (c) Capacitance currents (caused by charging/discharging of the electric double layer) decrease faster with decreasing electrode area than faradaic currents do, so that the signal/noise ratio in voltammetry is greater at microelectrodes than at macroelectrodes, particularly at the fast potential sweep rates necessary to study fast heterogeneous or homogeneous processes (ref. 17, 18). (d) Diffusion profiles at microelectrodes differ from those at macroelectrodes in that radial diffusion (the "edge effect") is more important. This improved mass transfer has important consequences. First, the shapes of voltammograms obtained in potential sweep experiments at microelectrodes may differ from those at macroelectrodes. In both cases the familiar sigmoid-shaped curves resulting from steady-state limiting currents obtained at slow sweep rates merge into peak-shaped curves at higher rates, but the transition occurs at higher rates with smaller electrodes. The advantage of steady-state currents is that they are easier to measure, thereby facilitating kinetic studies. Second, certain complicating homogeneous processes (e.g., following chemical reactions) coupled to electrode reactions have less effect than at macroelectrodes, thereby simplifying studies of such electron transfer processes and/or allowing studies of faster processes (ref. 19).

The main characteristics of microelectrodes will now be discussed more fully. 


\section{The steady-state diffusion current}

It was shown as early as 1977 by Berg and Purcell (ref. 20) that the flux of molecules diffusing into a biological cell is given by eq (6), where a is the radius of the cell and

$$
J=4 \pi a D C *
$$

$D$ and $C^{*}$ are the diffusion coefficient and the concentration of the molecules. It is to be noted that, for the small values of a applying here, $J$ is proportional to the diameter and not to the area of the cell. The electrical analog of eq (6) is eq (7), where $i_{d}$ is the

$$
i_{\mathrm{d}}=4 \pi \mathrm{nFCDC} *
$$

diffusion current and $C$ is capacitance. Solutions of eq (7) are available for a variety of conductors. For an isolated conducting disc of radius $r$ lying in an insulating plane, $C=2 r / \pi$, so that eq (8) applies.

$$
i_{\mathrm{d}}=4 \mathrm{nFrDC} *
$$

It is instructive to consider in a more general way the influence of the size of the electrode on the current. The smaller the electrode is, the more closely the diffusion field approaches spherical symmetry (ref. 19). For a potential step to the diffusion plateau at a spherical electrode of any radius $r_{s}$, the current as a function of radius and time $t$ is given by eq (9), where the first term represents the transient component which is

$$
i=4 \pi n F C *\left\{\left(r_{s}^{2} D^{1 / 2} / \pi 1 / 2 t^{1 / 2}\right)+r_{s} D\right\}
$$

proportional to the area of the electrode (the familiar Cottrell equation) while the second term gives the time-independent steady-state component which is proportional to the radius (not the area) of the electrode. The important consequence is that the steadystate component predominates at sufficiently long times for an electrode of any size but that this begins to occur at shorter times for smaller electrodes. That this prediction is borne out in practice is shown by the results illustrated in Fig. 3 .
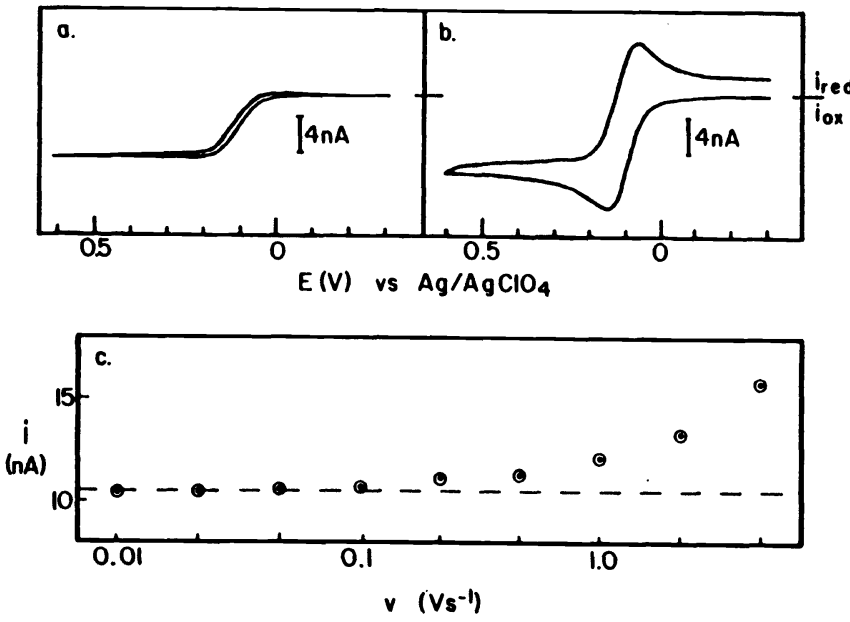

Figure 3

Cyclic voltammograms for the oxidation of ferrocene in acetonitrile at a gold microelect rode of $6.5-\mu \mathrm{m}$ radius. (a) Scan rate $0.1 \mathrm{~V} \mathrm{~s}^{-1}$. (b) Scan rate $10 \mathrm{~V} \mathrm{~s}^{-1}$. (c) Maximum current as a function of scan rate for $2.25 \mathrm{mM}$ ferrocene. It is to be noted that at a macroelectrode the signal will be as in (b) even for conditions as in (a). From ref. (18), by permission.

\section{The capacitance current}

In voltammetry, the faradaic current is the signal while the capacitance current is a component of the noise. For a potential pulse of amplitude $\Delta E$, the capacitance current flowing as a result of electric double layer charging/discharging is given by eq (10),

$$
1_{c}=(\Delta E / R) \exp (-t / R C)
$$

where $R$ is the total resistance of the cell, $C$ is the double layer capacitance, and $t$ is the time elapsed after application of the pulse. The capacitance is directly proportional to the electrode area, while for a microdisc the resistance varies inversely with electrode radius ( $n o t$ area) as $R=\rho / 4 r$, where $\rho$ is the resistivity of the solution (ref. 21). It follows from eq (9) and (10) that the capacitance current decreases proportionately more with decreasing electrode radius than the faradaic current does. Consequently, the signal/noise ratio should increase for all times $t$ with decreasing 
electrode radius (although instrumental and other problems may negate this potential advantage). Alternatively, acceptable signal/noise ratios can be maintained at shorter times $t$ by using smaller electrodes, thereby allowing "ultrafast" voltammetry, as shown in Fig. 4. Such measurements make it possible to determine the rates of very fast processes.

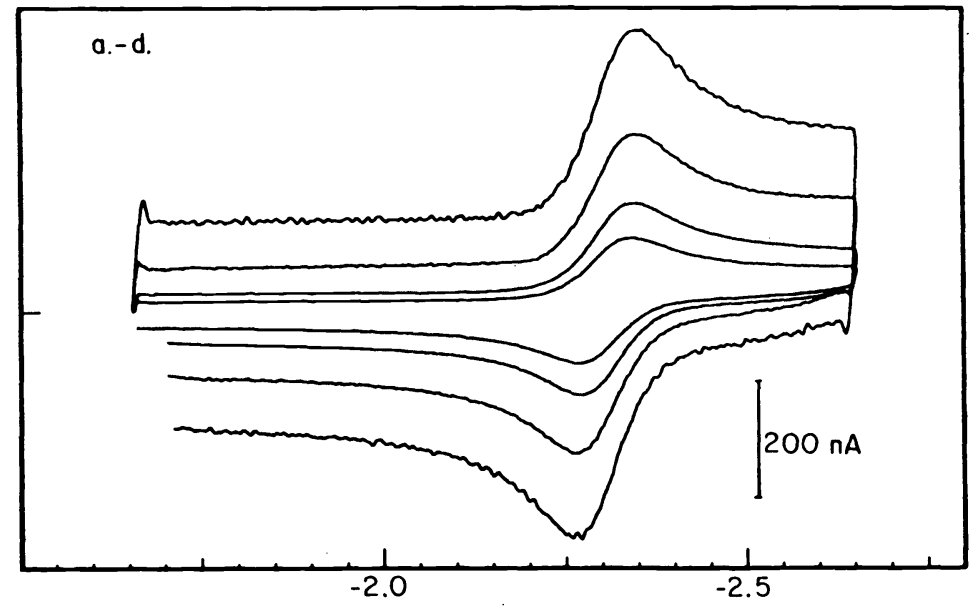

$E(V)$ vs $\mathrm{Ag} / \mathrm{AgClO}_{4}$

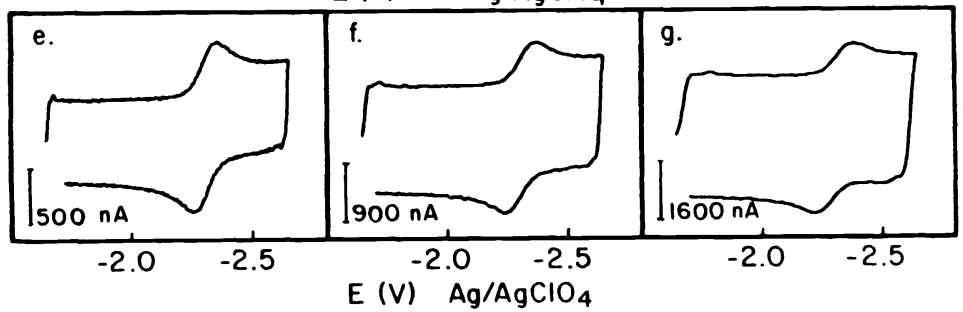

Figure 4

Cyclic voltammograms for the reduction of anthracene in acetonitrile at a gold microelectrode of $6.5-\mu \mathrm{m}$ radius. Scan rates $\left(\mathrm{V} \mathrm{s}^{-1}\right):$ (a) 1000 , (b) 2000 , (c) 5000 (d) 10,000 , (e) 20,000 , (f) 50,000 , and (g) 100,000. From ref. (18), by permission.

For example, a cyclic voltammogram will be reversible at a given scan rate $\nu\left(V \mathrm{~s}^{-1}\right)$ if the half-life (s) of an electrogenerated intermediate is greater than $10^{-2} / v$ (ref. 22). Consequently, in order to intercept intermediates with half-lives in the microsecond range, scan rates of the order of $10^{4} \mathrm{~V} \mathrm{~s}^{-1}$ are required. Wightman et al. (ref. 22) have employed such scan rates at microelectrodes to measure standard oxidation potentials of alkyl-substituted benzenes in trifluoroacetic acid as solvent. These potentials were found to correlate well with vertical ionization potentials measured in the gas phase by photoelectron spectroscopy.

\section{The potential drop in highly resistive solutions}

In all controlled potential methods, any uncompensated solution resistance introduces an error because the effective potential is less (numerically) than the applied potential. A further limitation is that the cell response is slow due to a finite cell time constant. Various approaches have been followed to minimize these problems. Among the latest instrumental methods is intelligent, automatic compensation of solution resistance by means of a cybernetic potentiostat (ref. 23). In this way cyclic voltammetry of ferrocene in methylene chloride (permittivity $=9$ ) with $0.1 \mathrm{M}$ tetrabutylammonium tetrafluoroborate as supporting electrolyte could be much improved, with the anodic-to-cathodic peak separation decreasing from $224 \mathrm{mV}$ with no compensation to $59 \mathrm{mV}$ (as expected for a reversible one-electron process) with full compensation. In more resistive media, however, such instrumental compensation is not feasible.

At sufficiently small microelectrodes the iR drop is negligible even in such highly resistive solutions as acetonitrile with little or no supporting electrolyte added, or toluene with supporting electrolyte. In fact, most recently Murray et al. (ref. 16) extended such measurements to the nonpolar solvent hexane. Finding a suitable supporting electrolyte for hexane was a problem, however. Of many tested, the most effective was an equimolar $(0.2 \mathrm{M}) \mathrm{mixture}$ of dinonylnaphthalene sulfonic acid and trioctylphosphine oxide. This medium does have certain limitations. The first is that the electrochemical window of the supporting electrolyte is only $0.9 \mathrm{~V}$ wide, but it does allow voltammetry at currents up to ca. $500 \mathrm{pA}$ of such substances as ferrocene, either as such or as a film of poly(vinylferrocene). The second limitation is that the mole fraction and especially the volume fraction of the supporting electrolyte are by no means negligible as compared to corresponding quantities for the solvent. In polar solvents such problems can be avoided. 
While a supporting electrolyte is generally desirable for voltammetry at macroelectrodes (in order to minimize the migration current and the iR drop), it does create complications in that comparison of the results with those obtained by such methods as potentiometry and especially spectroscopy becomes tenuous. Furthermore, the supporting electrolyte necessarily introduces impurities that may interfere in both obvious and more insidious ways.

Applications of microelectrodes in solutions containing little or no supporting electrolyte have been reported by Bond et al. (ref. 14). The model process chosen for this work was the oxidation of ferrocene to ferricinium ion which in theory represents a one-ion problem in that a cation is generated without an anion being available. The solvent was acetonitrile which had been pretreated with alumina. Results are shown in Fig. 5 and 6.
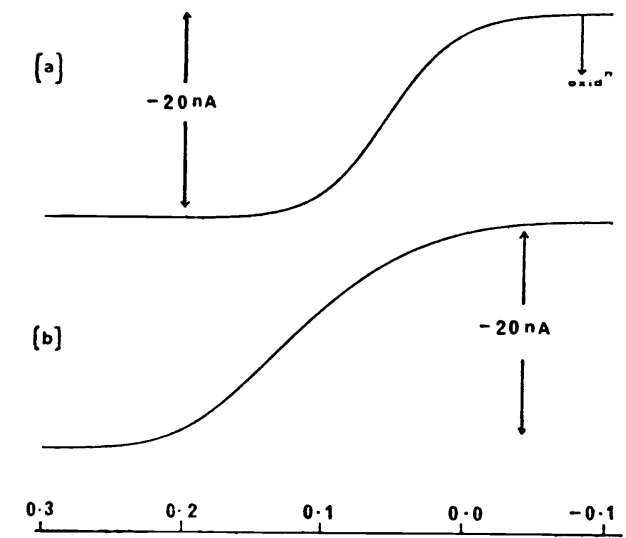

v. vs. $\mathrm{Ag} / \mathrm{Ag}^{+}$

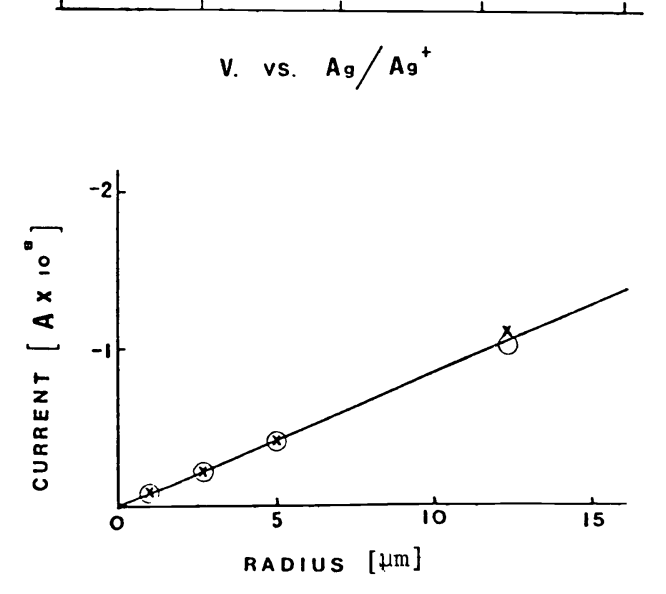

Figure 5

Cyclic voltammogram (scan rate $5 \mathrm{mV} \mathrm{s}^{-1}$ ) for the oxidation of $1 \mathrm{mM}$ ferrocene in acetonitrile at a platinum microelectrode of $25-\mu \mathrm{m}$ radius. (a) $0.1 \mathrm{M} \mathrm{Et}_{4} \mathrm{NClO}_{4}$ added. (b) No supporting electrolyte added. It is to be noted that smaller microelectrodes (for which the iR drop is smaller) give less drawn-out curves than (b). Difference in half-wave potential is probably caused by difference in 1iquid junction potential. From ref. (14), by permission.
Figure 6

Limiting current in cyclic voltammetry (scan rate $5 \mathrm{mV} \mathrm{s}^{-1}$ ) vs. radius for the oxidation of $1 \mathrm{mM}$ ferrocene in acetonitrile. Circles: $0.1 \mathrm{M} \mathrm{Et} 4 \mathrm{NC1O}_{4}$ added. Crosses: no supporting electrolyte added. From ref. (14), by permission.

It is to be noted that acetonitrile treated with alumina still contains low but nevertheless significant concentrations of such impurities as water, n-propylamine and acetic acid. Possible effects of residual water were discussed by Bond et al. We belleve that the other two impurities listed and also as yet unidentified ionic impurities are more significant in the present context. While proton transfer from a typical carboxylic acid to an amine should be negligible in acetonitrile (ref. 24), the electric conductivity of typically purified acetonitrile $\left(5-10 \times 10^{-8} \mathrm{ohm}^{-1} \mathrm{~cm}^{-1}\right)$ indicates that considerable concentrations of ionic impurities are present.

\section{Fast homogeneous processes coupled to electrode reactions}

As illustrations of the advantages of microelectrodes in studies of fast homogeneous processes coupled to electrode reactions, Fleischmann et al. (ref. 19) investigated typical $\mathrm{CE}$ (chemical reaction followed by electrochemical reaction; example: electrochemical reduction of acetic acid in water), EC' (electrochemical reaction followed by catalytic chemical reaction; example: electrochemical oxidation of ferrocyanide ion in presence of amidopyrine) and other processes. It was shown that the steady-state component of the current density at a microdisc electrode of radius $r_{d}$ is the same as that at a spherical electrode of radius $r_{s}$ equal to $\pi r_{d} / 4$. Fleischmann based his analysis on this analogy. More involved coupled processes were also treated. An example is the following reaction sequence of ten encountered in organic electrochemistry. 


$\begin{array}{ll}A \pm \mathrm{n}_{1} \mathrm{e} & \text { (a) } \\ \mathrm{B} & \mathrm{k}_{1} \\ \mathrm{C} \pm \mathrm{C} & \text { (b) } \\ \mathrm{n} 2 \mathrm{\textrm {n } _ { 2 }} \neq \mathrm{D} & \text { (c) } \\ \mathrm{B}+\mathrm{C} \neq \mathrm{\textrm {k } _ { 2 }} & \text { (d) }\end{array}$

Here, homogeneous electron transfer (step d) competes with heterogeneous electron transfer (step c). Three limiting cases of this sequence have been identified by Savéant (ref. 25): (i) Step d unimportant, step b rate determining (ECE process); (ii) step c unimportant, step b rate determining (disproportionation, DISP 1); and (iii) step c unimportant, step d rate determining (DISP 2). Most common electrochemical methods cannot distinguish clearly between ECE and DISP 1 mechanisms, but steady-state measurements at microelectrodes can do so. It can be shown that the apparent number of electrons involved in the ECE process shown is given by eq (11) while for a common DISP 1 process in which

$$
n_{a p p}=\frac{n_{2} r_{s}\left(k_{1} / D_{B}\right)^{1 / 2}}{1+r_{s}\left(k_{1} / D_{B}\right)^{1 / 2}}+n_{1}
$$

both chemical steps are irreversible it is given by eq (12), where all relevant diffusion

$$
\left.\mathrm{n}_{\mathrm{app}}=2 \mathrm{n}\right\} \frac{1+\mathrm{r}_{\mathrm{s}}\left(2 \mathrm{k}_{1} / \mathrm{D}\right)^{1 / 2}}{2+\mathrm{r}_{\mathrm{s}}\left(2 \mathrm{k}_{1} / \mathrm{D}\right)^{1 / 2}}
$$

coefficients $D$ are assumed to be equal. For the special case when $n_{1}=n_{2}=1$, $n_{\text {app }}$ should vary from a limiting value of 2 for very large electrodes to one of 1 for very small electrodes. These predictions were verified for two cases: (i) 0xidation of anthracene in acetonitrile $\left(E_{r} C_{1} E_{r}\right.$ process; $r$ : reversible, 1 : irreversible). It was found that water behaves as such a strong nucleophile that nucleophilic attack by acetonitrile does not occur unless the solvent is "superdry" (trifluoroacetic acid added). (ii) Oxidation of hexamethylbenzene in acetonitrile (DISP 1 process).

\section{Application to chronoamperometry, chronopotentiometry and potentiometric stripping analysis}

The techniques listed here also benefit from the use of microelectrodes for the same reasons as discussed before. The theory of chronoamperometry was treated by Shoup and Szabo (ref. 26) for the case of a stationary microdisc lying in an infinite insulating plane by using both analytical and digital simulation techniques. It was shown that, while the current does decay as $t^{-1 / 2}$ to a steady-state value, it does so with an amplitude differing by a factor of $8 / \pi^{2}$ from that predicted by the Cottrell equation.

The theory of chronopotentiometry at a stationary microdisc electrode was presented by Aoki et al. (ref. 27). Equations were given for the potential-time curve as well as for the transition time as a function of the ratio $i_{s s} / 1$ where $i_{s s}$ is the steady-state diffusion current and $i$ is the constant current applied in the chronopotentiometric experiment.

Potentiometric stripping analysis (PSA) is a technique for ultratrace analysis. It resembles conventional voltammetric stripping analysis (VSA) in that preconcentration of the analyte (metal ion) from solutions as dilute as $10^{-10} \mathrm{M}$ is accomplished by electrodeposition in a thin film of mercury, but it differs from VSA in that the metal analgam is subsequently oxidized ("stripped") chemically, rather than electrochemically. We have shown (ref. 28) that PSA is applicable not only to noble metals but also to highly electropositive elements. For example, sodium and potassium ions could be determined in such samples as seawater and blood serum after adding a large excess of dimethyl sulfoxide, which slows down premature oxidation of the amalgam by the water present, as shown in Fig. 7. In this work, macroelectrodes consisting of thin films of mercury on glassy carbon substrates were used. Here, again, microelectrodes offer demonstrated or potential advantages (ref. 29). For example, $E=f(t)$ transients are better defined at short times, and such interfering follow-up reactions as precipitation of alkali metal hydroxides on the electrode during stripping in aprotic solvents containing some water are minimized for reasons indicated before. Furthermore, resolution of transients for mixtures of analytes is improved. This advantage is predicted by the theory of PSA developed by Hussam and Coetzee (ref. 30), who based their treatment on an assumed initial parabolic concentration gradient of the metal amalgam as shown in Fig. 8. For a macroelectrode, the transient potential as a function of time is given by eq (13), where $\ell$ is the

$$
\left.E_{t}=E^{\circ}-\frac{R T}{n F} \text { ln }\left\{n\left(\frac{\pi D \tau}{2 \ell^{2}}\right)^{1 / 2}\right\}-\frac{R T}{n F} \ln \mid \frac{1-\phi}{\phi^{1 / 2}}\right\}
$$




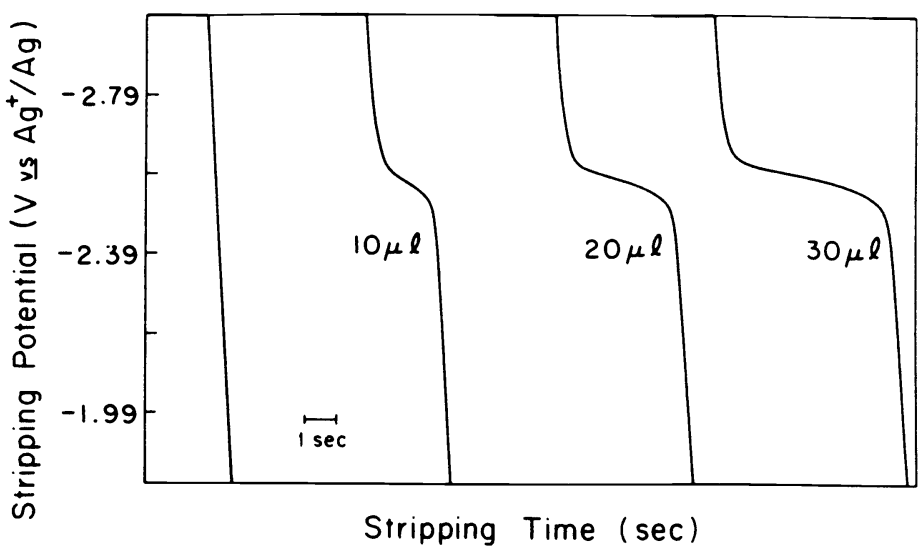

Hg-Film Diffusion Layer Bulk
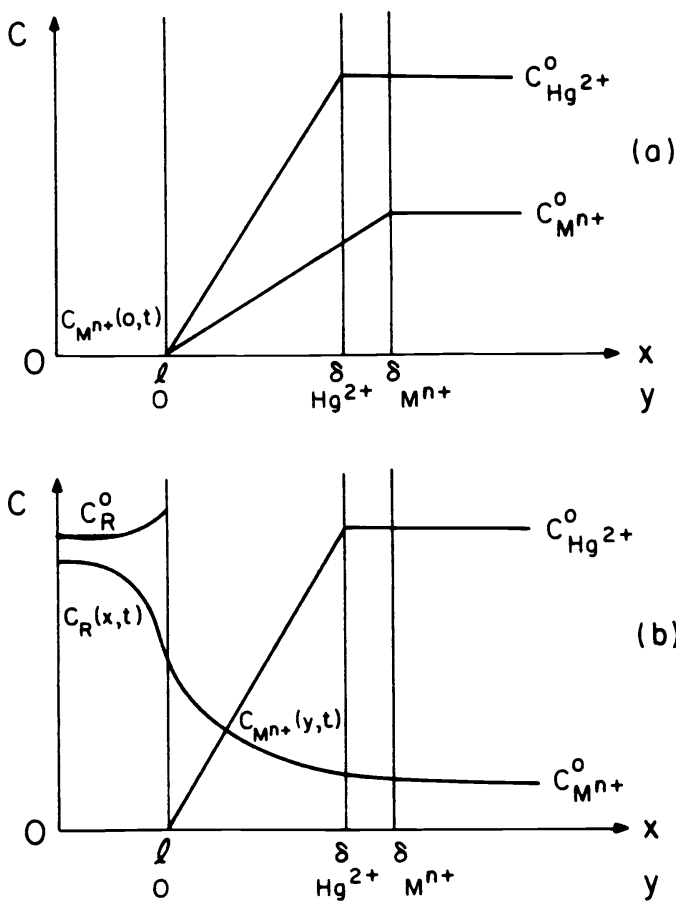

Figure 7

Potentiometric stripping signals of human blood serum in dimethy 1 sulfoxide. Conditions: successive injection of $10-\mu \mathrm{L}$ aliquots of serum into $20 \mathrm{~mL}$ of $10^{-3} \mathrm{M}$ $\mathrm{HgCl}_{2}+10^{-1} \mathrm{M} \mathrm{Et}_{4} \mathrm{NClO}_{4}$. From ref. (28), by permission.
Figure 8

Assumed concentration profiles during (a) potentiostatic deposition of metal ion $\mathrm{M}^{\mathrm{n}+}$, and (b) potentiometric stripping of the amalgam $R$ with $\mathrm{Hg}^{2+}$. From ref. (30) by permission.

thickness of the mercury film, $\tau$ is the transition time and $\phi=t / \tau$. For a microelectrode, on the other hand, the time dependence of the potential is different, and eq (14) applies, where $\delta$ is the diffusion layer thickness. Hence, the initial drop in the

$$
E_{t}=E^{\circ}-\frac{R T}{n F} \ln \left(\frac{D \tau}{\delta l}\right)-\frac{R T}{n F} \ln (1-\phi)
$$

transient potential occurs faster at a microelectrode than at a macroelectrode, giving a better defined (flatter) transient.

\section{Conclusions}

We have shown in this section that microelectrodes have significant advantages in a variety of electrochemical techniques. These advantages are particularly important for nonaqueous solutions. 


\section{OTHER TRENDS AND POTENTIALITIES}

In this section only a brief outline is given of additional areas in which the electrochemistry of nonaqueous solutions already is important or holds significant promise.

\section{New solvents and electrolytes}

There appears to be a hiatus in the introduction of new polar liquids suitable as solvents for conventional electrochemistry, but new solid-state electrolytes (ionic conductors) are regularly becoming available (ref. 31, 32). Such electrolytes are finding increasing use, especially in storage batteries. Examples are conducting polymer-alkali metal complexes and vitreous electrolytes (conducting glasses). The electric conductivities of certain glasses are similar to maximum values attainable in such highly conducting organic solvents as dimethoxyethane. A typical high energy density battery in which a conducting glass is used is $\mathrm{Li} / 2.5 \mathrm{LiI} \cdot \mathrm{Li}_{4} \mathrm{P}_{2} \mathrm{~S}_{7} / \mathrm{TiS}_{2}$ which can provide a continuous discharge current density of $2 \mu \mathrm{A} \mathrm{cm}^{-2}$ at $-40{ }^{\circ} \mathrm{C}$ and more than $800 \mu \mathrm{A} \mathrm{cm}-2$ at $+150{ }^{\circ} \mathrm{C}$ ( $\mathrm{J}$. R. Akridge in ref. 32). Such batteries have long shelf life and good high-temperature capability, but suffer from low current (not energy) densities and such manufacturing problems as extreme sensitivity to water and difficulties in ensuring adequate contact between the electrodes and the electrolyte. One of the most useful electrochemical techniques available for studies of solid-state (and liquid-state) batteries is complex plane impedance analysis (ref. 32).

Attempts to improve liquid-state lithium batteries are continuing unabated (ref. 4 ). Significant problems remain even for primary batteries in which such extensively studied solvents as propylene carbonate and thionyl chloride are used, the latter serving as both solvent and cathode. Analytical problem solving in battery research and development was discussed by Blomgren (ref. 33) at the 1984 meeting of this Conference. Among the important unanswered questions at this time is how the desolvation rate of lithium ion affects battery performance. The applicability of standard electrochemical techniques to such studies is illustrated by the work of Jorné and Pai (ref. 34).

Advances in molten salt electrochemistry will not be discussed here.

Super-critical fluids offer interesting possibilities for electrochemistry (as well as for chromatography). These fluids differ from ordinary liquids in that they have lower densities, viscosities and permittivities as well as different solvation properties. Bard et a1. (ref. 35) have shown that standard electrochemical techniques can be applied to nearcritical and super-critical ammonia (critical point $133^{\circ} \mathrm{C}$ and 112.5 bar). For example, useful cyclic voltammograms were obtained for the generation and the oxidation of solvated electrons and for the reduction of m-chloronitrobenzene.

\section{Chemically modified electrodes}

The purpose of chemical modification of electrodes is to channel electrode reactions into desired pathways. Such modification is accomplished by covalent anchoring of polymers containing desired moleties and by other methods. There is much activity in this field; a particularly useful introductory review has been presented by Faulkner (ref. 36). Increasing applications to nonaqueous solutions for such purposes as electrocatalysis and stabilization of radicals are to be expected.

\section{Semiconductors as electrodes}

The accessible potential range of certain solvents can be extended by using semiconductor electrodes. Kohl and Bard (ref. 37) studied the behavior of such semiconductors as silicon and n-type $\mathrm{ZnO}$, $\mathrm{CdS}$ and $\mathrm{GaP}$ in acetonitrile, chosen because its accessible potential range (i.e., the effective solvent band gap) is wide. Thus, oxidation or reduction via the conduction band of species with standard potentials beyond the flat-band potential could be carried out. It was observed that the anodic limit of $n-C d S$ (in the dark) in acetonitrile, which is determined by oxidation of CdS to cadmium ion, is above $+5 \mathrm{~V}$ vs. $\operatorname{SCE}(\mathrm{aq})$, as compared to a limit near $+2.5 \mathrm{~V}$ at a platinum electrode. The weak solvation of cadmium ion by acetonitrile contributes to this highly positive anodic limit. It appears that use can be made of such extensions in the electrochemical "window" of solvents.

\section{Transport properties of electrolytes in nonaqueous solutions}

Transport properties of nonaqueous solutions were thoroughly treated at the 1984 meeting of this Conference, when Chemla et al. (ref. 38) discussed the influence of preferential solvation in mixed solvents on the transport properties of electrolytes as measured by a useful radiotracer technique, and Barthel (ref. 39) discussed the temperature and con- 
centration dependence of transport properties as well as the detailed theoretical treatment of both dilute and concentrated solutions, the latter treated on the basis of ion distribution functions.

The dielectric properties of solutions are also of interest here. Recent developments include measurement of the dielectric properties of solutions of various $1: 1$ as well as hydrated 2:1 and 3:1 electrolytes in ethanol, 1-propanol and 1-hexanol (ref. 40). While all electrolytes studied increase the mean relaxation time in all solvents, their influence on the static permittivity varies markedly in magnitude and even in sign. The results were interpreted in terms of ion pair formation, the nature of the solvation shells and differences in structuring influences of the solutes.

A useful extension in the measurement of transference numbers to concentrated aqueous solutions was reported by Spiro et al. (ref. 41). By employing chlorine electrodes in cells with transference, as in $\mathrm{Ir}, \mathrm{Pt}\left|\mathrm{Cl}_{2}+\mathrm{N}_{2}\right| \mathrm{MCl}(\mathrm{m}) \mid \mathrm{MCl}\left(\right.$ ref. $\left._{0}\right)\left|\mathrm{N}_{2}+\mathrm{Cl}_{2}\right| \mathrm{Pt}$, Ir transference numbers of sodium ion were measured over the range 1.7 to 6 molal sodium chloride. This approach has advantages over the Hittorf method which is tedious and the moving boundary method which suffers from Soret effects in concentrated solutions. While it is generally more difficult to find potentiometric sensors that respond reversibly to appropriate ions over wide concentration ranges in nonaqueous (particularly aprotic) solutions, this approach is undoubtedly worth pursuing because transference numbers for concentrated nonaqueous solutions are needed for both fundamental reasons and such applications as nonaqueous batteries.

\section{Interfaces between immiscible solvents}

The study of solid-gas and solid-liquid interfaces is a field of intense current activity. The introduction of a wide array of such complementary spectroscopic techniques as photoelectron, Auger, electron energy loss, reflectance Fourier transform infrared and surfaceenhanced Raman spectroscopy, as well as secondary ion mass spectrometry, has revolutionized the investigation of fundamental processes occurring at surfaces, particularly in catalysis.

It has been known for a long time that interfacial phenomena at electrode surfaces of ten decisively influence electron transfer processes, and electrochemical and spectroscopic studies of such phenomena are continuing unabated. A much more recent development is the study of liquid-liquid interfaces, e.g., nitrobenzene-water. Such electrochemical techniques as cyclic voltammetry and complex plane impedance measurements can provide fundamental thermodynamic and kinetic information on the transfer of small molecules across the interface and on the adsorption of surface-active molecules at the interface. The characteristics of liquid-liquid interfaces are obviously relevant to such processes as solvent extraction; they also have features in common with those of electrode-solution interfaces and, as first suggested by Koryta, they may serve as a simple model for half of a biological membrane in contact with an electrolyte solution. An authoritative review of this novel field has been given by Buck (ref. 42). It seems likely that further studies will lead to improved understanding not only of interfacial phenomena but also of interactions occurring in bulk solution phases.

\section{CONCLUSIONS}

In this paper certain current trends in electrochemical studies of nonaqueous solutions were discussed. An attempt was made to identify recent electrochemical approaches that hold particular promise for the development of nonaqueous solution chemistry. It is important to maintain a proper perspective, however. Interactions occurring in liquid solutions are so complex that their understanding will be best served by utilizing the full arsenal of experimental and theoretical approaches applicable to the study of solutions.

\section{Acknowledgements}

The author acknowledges the contributions of his coworkers in areas covered in this paper: T.-H. Chang, B. K. Deshmukh, M. Ecoff, C.-C. Liao and J. L. Vaughn. Financial support was provided by the National Science Foundation.

\section{REFERENCES}

1. J. F. Coetzee and R. J. Bertozzi, Anal. Chem. 43, 961 (1971).

2. B. K. Deshmukh and J. F. Coetzee, Anal. Chem. 56, 2373 (1984). 
3. E. Pungor, K. Tóth, P. G. Klatsmányi and K. Izutsu, Pure \& Appl. Chem. 55, 2029-2065 (1983).

4. J. P. Gabano, Ed., Lithium Batteries, Academic Press, New York (1983).

5. S. Pons and S. B. Khoo, Electrochim. Acta 27, 1161 (1982).

6. J. F. Coetzee, C.-H. Liao and T.-H. Chang, unpublished results (1986).

7. S. Gottesfeld and J. D. E. McIntyre, J. Electrochem. Soc. 126, 742 (1979).

8. J. D. E. McIntyre, W. F. Peck, Jr., and S. Nakahara, J. Electrochem. Soc. 127, 1264 (1980).

9. N. F. de Roolj and P. Bergveld, Monitoring of Vital Parameters during Extracorporeal Circulation, Proc. Int. Conf. Nijmegen 1980, pp. 156-165, Karger, Basel (1981).

10. T. Katsube, I. Lauks and J. N. Zeme1, Sensors \& Actuators 2, 399 (1982).

11. A. Fog and R. P. Buck, Sensors \& Actuators 5, 137 (1984).

12. S. Bordi, M. Carla and G. Papesch1, Anal. Chem. 56, 317 (1984).

13. T. E. Edmonds, Anal. Chim. Acta 175, $1-22$ (1985).

14. A. M. Bond, M. Fleischmann and J. Robinson, J. Electroanal. Chem. 168, 299 (1984).

15. R. M. Wightman, Anal. Chem. 53, 1125A-1134A (1981).

16. L. Geng, A. G. Ewing, J. C. Jernigan and R. W. Murray, Ana1. Chem. 58, 852 (1986).

17. J. 0. Howel1 and R. M. Wightman, J. Phys. Chem. 88,3915 (1984).

18. J. 0. Howe11 and R. M. Wightman, Anal. Chem. 56, 524 (1984).

19. M. Fleischmann, P. Lasserre and J. Robinson, J. Electroanal. Chem. 177, 115 (1984).

20. H. C. Berg and E. M. Purcel1, Biophys. J. 20, 193 (1977).

21. J. J. Newman, J. Electrochem. Soc. 113, 501 (1966).

22. J. 0. Howe11, J. M. Goncalves, C. Amatore, L. Klasinc, R. M. Wightman and J. K. Kochi, J. Am. Chem. Soc. 106, 3968 (1984).

23. P. He and L. R. Faulkner, Ana1. Chem. 58, 517 (1986).

24. J. F. Coetzee, in A. Streitwieser, Jr., and R. W. Taft, Eds., Progress in Physical Organic Chemistry, Vo1. 4, pp. 45-92, Wiley, New York (1967).

25. C. Amatore and J. M. Saveant, J. Electroanal. Chem. 85, 27 (1977).

26. D. Shoup and A. Szabo, J. Electroana1. Chem. 140, 237 (1982).

27. K. Aoki, K. Akimoto, K. Tokuda, H. Matsuda and J. Osteryoung, J. Electroanal. Chem. 182, 281 (1985).

28. J. F. Coetzee, A. Hussam and T. R. Petrick, Anal. Chem. 55, 120 (1983).

29. J. F. Coetzee and $M$. Ecoff, unpublished results.

30. A. Hussam and J. F. Coetzee, Anal. Chem. 57, 581 (1985).

31. D. F. Shriver and G. C. Farrington, Chem. \& Eng. News, May 20, 1985, pp. 42-57.

32. J. B. Boyce, L. C. De Jonghe and R. A. Huggins, Eds., Solid State Ionics-85, Proc. 5th International Conf. on Solid State Ionics, Parts I and II, North-Holland (1985).

33. G. E. Blomgren, Pure \& App1. Chem. 57, 383-388 (1985).

34. J. Jorné and P. G. Pai, J. Electrochem. Soc. 132, 1612 (1985).

35. R. M. Crooks, F.-R. F. Fan and A. J. Bard, J. Am. Chem. Soc. 106, 6851 (1984).

36. L. R. Faulkner, Chem. \& Eng. News, Feb. 27, 1984, pp. 28-45.

37. P. A. Koh1 and A. J. Bard, J. Am. Chem. Soc. 99, 7531 (1977).

38. M. Chemla, J. M'Halla, M. Perie and P. Turq, Pure \& Appl. Chem. 57, 369-382 (1985).

39. J. Barthe1, Pure \& App1. Chem. 57, 355-367 (1985).

40. B. Gestblom and J. Sjöblom, J. Solution Chem. 15, 259 (1986).

41. D. K. Panopoulos, H. Kaneko and M. Spiro, J. Solution Chem. 15, 243 (1986).

42. P. Vanýsek and R. P. Buck, J. Electroanal. Chem• 163, 1 (1984). 